\title{
Cluster strong lensing: a new strategy for testing cosmology with simulations
}

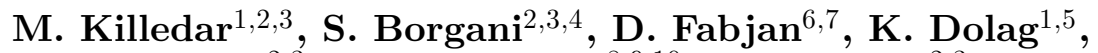 \\ G. Granato ${ }^{2,3}$ M. Meneghetti ${ }^{8,9,10}$, S. Planelles Pla $^{2,3}$ and \\ C. Ragone-Figueroa ${ }^{3,11}$ \\ ${ }^{1}$ Universitäts-Sternwarte München, Scheinerstrasse 1, D-81679, München, Germany \\ e-mail: killedar@usm.lmu.de \\ ${ }^{2}$ Astronomy Unit, Department of Physics, University of Trieste, Via Tiepolo 11, I-34131 \\ Trieste, Italy \\ ${ }^{3}$ INAF - Osservatorio Astronomico di Trieste, Via G.B. Tiepolo 11, I-34131 Trieste, Italy \\ ${ }^{4}$ INFN - National Institute for Nuclear Physics, Via Valerio 2, I-34127 Trieste, Italy \\ ${ }^{5}$ Max-Planck-Institut für Astrophysik, Garching, Germany \\ ${ }^{6}$ SPACE-SI, Slovenian Centre of Excellence for Space Sciences and Technologies, Aškerčeva 12, \\ 1000 Ljubljana, Slovenia \\ ${ }^{7}$ Faculty of Mathematics and Physics, University of Ljubljana, Jadranska 19, 1000 Ljubljana, \\ Slovenia \\ ${ }^{8}$ INAF - Osservatorio Astronomico di Bologna, Via Ranzani 1, I-40127 Bologna, Italy \\ ${ }^{9}$ INFN - Sezione di Bologna, Viale Berti Pichat 6/2, I-40127 Bologna, Italy \\ ${ }^{10}$ Jet Propulsion Laboratory, 4800 Oak Grove Dr. Pasadena, CA 91109, USA \\ ${ }^{11}$ Instituto de Astronomía Teórica y Experimental (IATE), Consejo Nacional de \\ Investigaciones Científicas y Técnicas de la República Argentina (CONICET), Observatorio \\ Astronómico, Universidad Nacional de Córdoba, Laprida 854, X5000BGR, Córdoba, Argentina
}

\begin{abstract}
Comparisons between observed and predicted strong lensing properties of galaxy clusters have been used to claim either tension or consistency with $\Lambda$ CDM cosmology. However, standard approaches to such tests are unable to quantify the preference for one cosmology over another. We advocate a Bayesian approach whereby the parameters defining the scaling relation between Einstein radii and cluster mass are treated as the observables. We demonstrate a method of estimating the likelihood for observing these parameters under the $\Lambda$ CDM framework, using the X-ray selected $z>0.5$ MACS clusters as a case in point and employing both N-body and hydrodynamic simulations of clusters. We account for cluster lens triaxiality within the modelling of the likelihood function. Cluster selection criteria is found to play as important a role as the uncertainty related to the description of star formation and feedback.
\end{abstract}

Keywords. cosmology: miscellaneous, gravitational lensing, galaxies: clusters: general, methods: n-body simulations, methods: statistical, X-rays: galaxies: clusters

\section{Introduction}

Galaxy clusters gravitationally lens and distort the images of background galaxies; their lensing efficiency is a powerful probe of cosmology with the ability to constrain structure formation parameters. The earliest comparisons between simulated clusters and the observed frequency of arc-like lensed galaxy images in a cluster sample revealed an order of magnitude difference between the observations and $\Lambda$ CDM predictions (Bartelmann et al. (1998), Li et al. (2005)). This discrepancy was dubbed the 'arc-statistics problem', and now is part of a broader study of cluster concentrations and strong lensing efficiencies. 
In the present work, we take the well-studied $z>0.5$ MACS clusters as our case in point (Horesh et al. (2010), Meneghetti et al. (2011), Zitrin et al. (2011), Waizmann et al. (2014)). We propose a Bayesian approach to the strong lensing cosmological test, employing clusters modelled within $\Lambda$ CDM hydrodynamic simulations which include the effects of stellar and AGN feedback. Massive clusters have been modelled in four different flavours of smooth-particle hydrodynamic ( $\mathrm{SPH}$ ) simulations: with only dark matter $(D M)$; including non-radiative hydrodynamics $(N R)$; including cooling, star formation and supernova feedback $(C S F)$; and further including AGN feedback $(A G N)$. For more details on the simulations, see Planelles et al. (2014).

\section{2. $\Lambda$ CDM strong lensing likelihood}

Strong lensing efficiencies, as characterised by the Einstein radii, scale well with the mass of clusters at large overdensities (Killedar et al. (2012)). If the $z>0.5$ MACS sample are, in fact, stronger lenses than predicted by the $\Lambda$ CDM model, they will have larger Einstein radii for a given total mass at low overdensities (or a proxy thereof).

A Bayesian approach to cosmological parameter estimation is advocated, in which one determines the relative preference of two hypothetical choices of cosmological parameters, $C_{1}$ and $C_{2}$, in light of the data $D$, by calculating the likelihood ratios: $\mathcal{L}\left(D \mid C_{1}\right) / \mathcal{L}\left(D \mid C_{2}\right)$, and subsequently multiplying by their relative priors. The initial aim is to calculate the likelihood of observing the Einstein radii of the high-z MACS sample under one chosen hypothesis: $\Lambda \operatorname{CDM}\left\{\Omega_{\Lambda, 0}=0.76 ; \Omega_{\mathrm{M}, 0}=0.24 ; \Omega_{\mathrm{b}, 0}=0.04 ; h=0.72 ; \sigma_{8}=0.8 ; P(k) \propto k^{n}\right.$ with $n=0.96$, with the aid of mock samples from numerical simulations. The likelihood function related to the observables $\left(\theta_{E}\right.$ and $\left.M_{500}\right)$ is intractable because there are a finite number of objects from the simulations. Instead, we assume a power-law relation between the strong lensing and mass:

$$
\log \left[\frac{M_{500}}{9 \times 10^{14} M_{\odot}}\right]=\alpha \log \left[\frac{\theta_{E}}{20 "} \sqrt{\frac{D_{d}}{D_{d s}}}\right]+\beta,
$$

and use the MCMC sampler emcee (Foreman-Mackey et al. (2013)) to infer the joint posterior, $P(\alpha, \beta)$ while marginalising out the intrinsic scatter (Hogg et al. 2010). We average over the posteriors of many mock samples and re-interpret this as a 'likelihood function': the probability that one would observe the scaling relation $\{\alpha, \beta\}$ under the hypothesis that $\Lambda \mathrm{CDM}$ is the true description of cosmology. From the MACS clusters, we obtain the posterior $P(\alpha, \beta)$ which is interpreted as a single 'data point'. We calculate the likelihood, $\mathcal{L}$, of observing $\{\alpha, \beta\}$ by convolving the 'data-point' and 'likelihood function'.

Note that one cannot comment on whether the likelihood is large or small. One cannot use this value to claim 'consistency' or 'tension' with $\Lambda$ CDM. However, if the same process is repeated for simulations under a different cosmological model then the Bayes factor can be calculated and, after accounting for priors, it may (or may not) reveal a preference for one of the cosmologies, in light of this data.

\section{Cluster Selection}

In the left panel of Fig. 1 we show the relation between the Einstein radii and the cluster mass $\mathrm{M}_{500}$. Measuring the strong lensing likelihood using $z=0.5$ clusters from the $A G N$ simulations that exceed the X-ray flux threshold, we find: $\mathcal{L}=0.27$. 

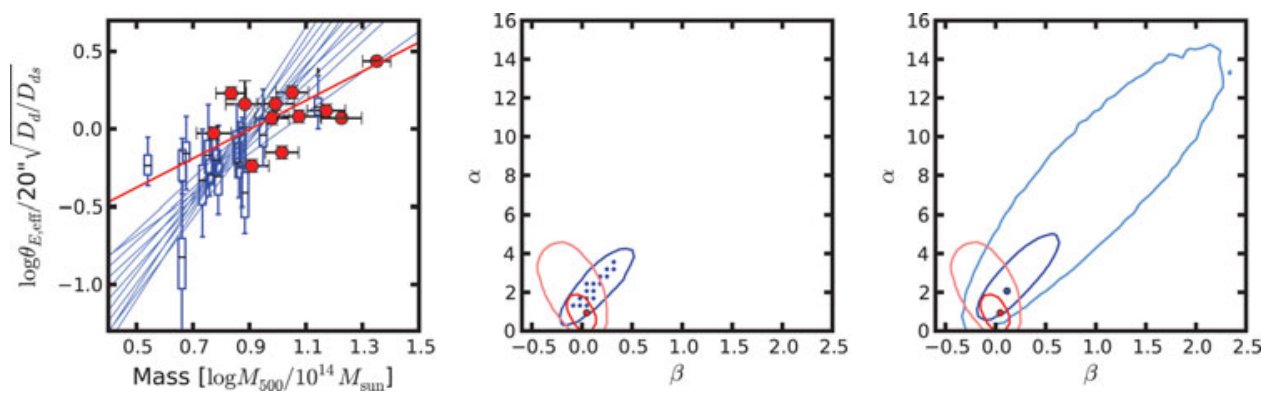

Figure 1. Left: Einstein radii, $\theta_{\mathrm{E}, \mathrm{eff}}$, plotted as a function of $M_{500}$. The range of Einstein radii for each $z=0.5$ cluster from the $A G N$ simulations is shown by a box-plot. The circles represent the MACS $z>0.5$ clusters (Mantz et al. (2010)). The thick line marks the maximum a-posteriori fit to observational data, while the thin lines mark the fit to 20 randomly chosen mock samples from simulations. Middle: 1- $\sigma$ and 2- $\sigma$ constraints on parameters of the strong lensing - mass relation given the MACS $z>0.5$ cluster data (contours), with a maximum a posteriori fit marked by a filled circle. Overplotted in dots are the best fits to 80 mock observations of the simulated cluster sample. A typical 1- $\sigma$ error is shown as an ellipse. Right: Constraints from the MACS $z>0.5$ cluster data are the same as in the middle panel, but the dark filled circle and curves mark respectively the maximum and the 1 and $2-\sigma$ contours of the likelihood function found by combining all 80 mocks.

We then consider how the measured likelihood for $\Lambda$ CDM may depend on other details, such as cluster selection criteria and the numerical implementation of hydrodynamics.

Selection by dynamical state: Consider now the effect of applying the incorrect selection criteria when modelling lenses. Excluding the most disturbed clusters, the likelihood function derived from simulations is more sharply peaked than that which is derived without this additional selection. If this relaxed simulated sample is used to analyse the full observational sample, one would incorrectly derive a likelihood of $\mathcal{L}=1.23$.

Selection by mass: Since the clusters in the MACS survey are selected by flux rather than luminosity, there is no corresponding mass threshold, yet it is common practise to select simulated clusters by mass for similar studies. We find that when selecting simulated clusters by mass (instead of flux), the likelihood increases to $\mathcal{L}=0.54$.

Hydrodynamics: We determine the sensitivity of our conclusions to the inclusion of baryonic processes and the resulting gas distribution. Using cluster counterparts from the $C S F$ simulations leads to $\mathcal{L}=1.28$, four times that derived from the $A G N$ simulations, while the $D M$ and $N R$ simulations result in $\mathcal{L}=0.16$ and 0.11 respectively.

\section{References}

Bartelmann, M., Huss, A., Colberg, J. M., Jenkins, A. , \& Pearce, F. R. 1998, A\&3 A, 330, 1 Foreman-Mackey D., Hogg D. W., Lang D., \& Goodman J. 2013, PASP, 125, 306

Hogg, D. W., Bovy, J., \& Lang, D. (2010), ArXiv: 1008.4686

Horesh, A., Maoz, D., Ebeling, H., Seidel, G., \& Bartelmann, M. 2010, MNRAS, 406, 1318

Killedar, M., Borgani, S., Meneghetti, M., Dolag, K., Fabjan, D., \& Tornatore, L. 2012, MNRAS, 427,533

Li, G.-L., Mao, S., Jing, Y. P., Bartelmann, M., Kang, X., \& Meneghetti, M. 2005, ApJ, 635, 795

Mantz, A., Allen, S. W., Ebeling, H., Rapetti, D., \& Drlica-Wagner, A. 2010, MNRAS, 406, 1773

Meneghetti, M., Fedeli, C., Zitrin, A., et al. 2011, A\&AA, 530, A17

Planelles S., Borgani S., Fabjan D. et al. 2014, MNRAS, 438, 195

Waizmann, J.-C., Redlich, M., Meneghetti, M., \& Bartelmann, M. 2014, A\&A, 565, A28

Zitrin, A., Broadhurst, T., Barkana, R., Rephaeli, Y., \& Benítez, N. 2011, MNRAS, 410, 1939 